\title{
QoS Management for Distributed Multimedia Services
}

\author{
Ralf Seepold, Natividad Martínez Madrid, and Javier Martínez Fernández \\ Departamento de Ingeniería Telemática \\ Universidad Carlos III de Madrid, Leganés (Madrid), Spain \\ \{ralf.seepold, natividad.martinez, javier.martinez\}@uc3m.es
}

\begin{abstract}
Currently, several multimedia devices support the UPnP protocol allowing automatic detection of devices, connection of devices and agreement on a certain quality of the communication established between a server of content (Media Server) and a player of content (Media Renderer). The focus of this paper is to add to the UPnP standard quality of service (QoS) some elements to incorporate better performance in the local networks. All these additions are compatible with the current standard.
\end{abstract}

\section{Introduction}

Nowadays, a wide variety of devices have in theory the capability to interact with each other. A solution for device interfacing is proposed by the UPnP (Universal Plug and Play) Forum [1]. With the help of the UPnP standard, new devices can be quickly detected, services can be used and devices are capable to start a direct communication. Within this group a specific architecture has been proposed that supports multimedia applications (UPnP AV architecture [2]) allowing for exchange of multimedia data from one device to another without any mediator.

For example, a trailer of a cinema movie offered by a Multimedia Server can be displayed in home-cinema equipment or on a mobile phone. Both so called Media Renderers have very different capabilities to display the video contents but from both of them it is expected that the video is shown in an acceptable quality. The crucial aspect remains: the provisioning of a service and fixing service quality that is required to enable the service in the dynamic environment of a network. This article proposes a solution that allows device integration into a home network, service registration in a home network platform and multimedia service delivery with Quality of Service (QoS) with a UPnP for a better performance in QoS management for local networks.

\section{Quality of Service Extension of the UPnP Standard}

The UPnP AV is directly linked to the UPnP QoS architecture [3], acting the audiovisual control point as the client of the QoS manager. The management of multimedia devices in a home network, and the distribution of the generated traffic with QoS have some requirements: 
1. Automatic discovery and configuration of multimedia devices

2. Request for establishment of traffic with QoS from the audiovisual control point to the QoS manager

3. Calculation of the path in a centralized or distributed way

4. Allowance of different mechanisms to specify the QoS (prioritized or parameterized)

5. Possibility of different admission control strategies: centralized (decided by the QoS manager), distributed (decided by the devices) or hybrid.

Only requirements 1 and 2 would be automatically guaranteed by using the UPnP AV and QoS architectures. The establishment of traffic with QoS is a stateless service. This means that the list of devices in the network is the only information kept by the QoS manager between two invocations of the service. All other information regarding topology of the network, characteristics of the network and the devices, or already admitted traffic flows, will be erased and recalculated for each invocation. While this strategy might have sense in a network with a very high degree of device mobility, the kind of home media networks addressed in this work are relatively stable, and therefore it makes sense to store different information regarding its state.

Furthermore, UPnP QoS V2 is only addressing the management of QoS for prioritized traffic, but there is no way to express other types of parameters (bandwidth, allowed delays, etc.) to administrate the resources.

The proposed extended functional model for the QoS architecture allows the QoS manager to store state information and to check it, having thus a better performance. As in the standard architecture, the service of establishment of traffic with QoS is requested by a client control point, typically audiovisual. The QoS Manager receives the request and checks first of all within the QoS Policy Holder the priority to be assigned to the traffic according to its policy rules. The diagram of the functional model is shown in Figure 1.

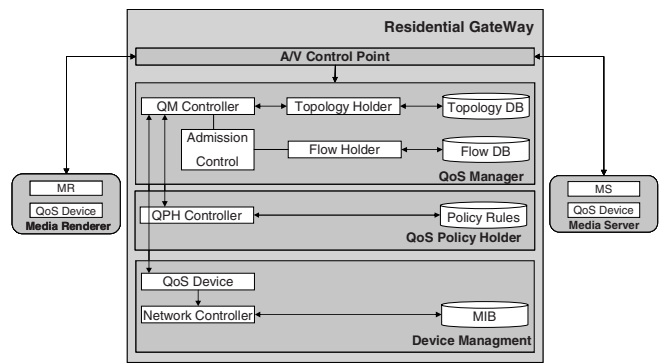

Fig. 1. Extended Functional Architecture for one Local Network

The QoS Manager is the main element, and is divided into four main modules:

The QoS Manager Controller receives the original request and orchestrates the interaction with the other internal and external components.

The QoS Topology Holder serves requests the paths between two devices in the network. In order to do this, it queries during setup the available multimedia devices and stores all information necessary to describe the topology of the network. After 
storing this information for each subsequent path query, it does not need to interact again with the devices, but just calculates the route according to the stored topology. All previously calculated paths are cached in a table to speed up the reply time. The topology and path table are kept stable between QoS requests, unless a device is added or removed. In this case, the tables should be recalculated.

The QoS Flow Holder gives support to the Admission Control component. It stores the dynamical information about the flows being transmitted at a given moment, including information about the path they are following, their priorities and parameters. In the current version of the standard, this information is not stored in any central place, so the manager has to query all devices in the path for their current state before admitting a new flow. This component would allow its flow querying and updating. Each time a new flow is admitted, an entry is added in the flow table. Correspondingly, each time a flow finishes, its entry is removed from the table. The first assumption is actually also made in the standard UPnP QoS architecture, and the second one is not considered to be too restrictive for the proposed scenario.

The QoS Admission Control component performs the traffic admission control, being therefore the main component of the manager. Its tasks include: Distribution and adaptation of QoS requirements along the traffic path, relevant for parameterized QoS; Orchestration of the admission control.and Decision in case of traffic rejection.

The Device Management module encapsulates the functionality of the residential gateway as an UPnP QoS Device, according to the standard service specification.

The scenario described is based on the standard architectures UPnP audiovisual (unmodified) and UPnP QoS (modified to be able to store state and take into account parameterized QoS). Both architectures assume that all devices are on the same local network. If the devices are located in different local networks connected though a residential gateway, the solution can be an easy variant of the previous scenario.

An AV control point in a single local network can only discover (and announce himself to) Media Servers and Media Renderers inside his own network. The same applies to the QoS Manager, which acts as control point of the QoS Devices. The proposed solution takes advantage of the fact that the residential gateway is a device connected to both networks, and thus provides both the audiovisual control point and the QoS Manager with the QoS Policy Holder. Figure 2 shows a communications module with the mentioned services and with a different functionality that the standard.
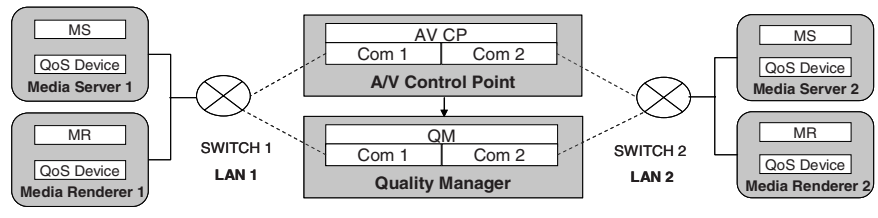

Fig. 2. Extended Functional Architecture for more than one local network

The QoS Manager will also perform its functionality in similar way to the one presented in the first scenario, except that it will have to add the network identifier to the device information, and that the residential gateway itself will always be on the path if the source and destination of the traffic are in different local networks. 


\section{Implementation and Conclusions}

The different scenarios have been tested with a gateway that offers a hardware platform running on Linux. The system was started on the embedded pc. On top of the operating system, an OSGi framework [4] (Oscar [5]) has been selected. This decision has been taken because we wanted to have a backup position when access to the source code is required. As a bundle (an OSGi application) an UPnP control point has been adopted and integrated into the OSGi framework. The Control Point of Cidero [6] has been selected for the test. The media server (CyberMediaGate [7]) has been installed as well in the gateway. Finally, a special bundle has been designed to launch both applications in the framework.

External to the gateway two configurable switches have been connected. These switches offer accessibility in the different home LANs. UPnP Renderers and UPnP Media Servers have been installed in computers running Windows, Linux or mobile devices like the Nokia N93. A Nokia 770 has been used as a remote control.

Now the QoS Manager can perform admission control. The extension of the traffic descriptor allows a fine grained tuning of the possible multimedia flows according on the device's capability (stored in the profile data and derived from the current load).The presented approach extends the current UPnP standard with features that provide establishment of traffic with QoS from the audiovisual control point to the QoS manager, calculation of the path in a centralized or distributed way, allowance of different mechanisms to specify the QoS (prioritized or parameterized), the possibility to enable different admission control strategies: centralized (decided by the QoS manager), distributed (decided by the devices) or hybrid.

Acknowledgments. The work has been partly funded by the projects PLANETS (MEDEA+ A306 funded by the Spanish Ministry of Industry; FIT-330220-2005-111), MUSE (IST-Program; MUSE-IST 026442) and MARTES (ITEA 04006, funded by the Spanish Ministry of Industry under FIT-340000-2006-166).

\section{References}

1. Universal Plug and Play (UPnP) Forum (June 2007), http://www.upnp.org/

2. Universal Plug and Play (UPnP) Forum, UPnP AV Architecture: 1, (June 2002), http://www.upnp.org/specs/av/UPnP-av-AVArchitecture-v1-20020622.pdf

3. Universal Plug and Play (UPnP) Forum, Quality of Service V 2.0 (October 2006), www.upnp.org/specs/qos/

4. Open Service Gateway Initiative (OSGi) Alliance (2007), http://www.osgi.org

5. Open Source OSGi framework implementation, Oscar (June 2007), http://forge.objectweb.org/projects/oscar/

6. Cidero Software Solutions for the Digital Home (2007), http://www.cidero.com

7. Reference Implementation of UPnP AV, Satoshi Konno, Tokyo, Japan (June 2007), http://www.cybergarage.org/net/cmgate/java/index.html 